\title{
Materials Science \& Engineering: From Past \& Present to the Future...
}

\author{
Manoj Gupta* \\ Department of Mechanical Engineering, National University of Singapore, Singapore
}

Received: February 24, 2014; Accepted: February 24, 2014; Published: February 25, 2014

*Corresponding author: Manoj Gupta, Department of Mechanical Engineering, National University of Singapore (NUS), Singapore, Tel: +65-6516 6358; Fax: +65-6779 1459, E-mail: mpegm@nus.edu.sg

\section{Editorial}

Materials science and engineering has transformed every aspect of our lives. New materials and methods to engineer them have facilitated rapid progress in technology. Materials engineering plays an important role in applications such as aerospace/space, automotive, construction/structures, electronics, biomedical, consumer electronics, medicine, energy systems etc., Variety of materials such as metals/alloys, ceramics, polymers/plastics, composites and their combinations are in the use. R\&D of materials and their engineering focusses towards: (i) materials synthesis: development of new materials, (ii) materials processing: innovative ways to process materials into useful applications, (iii) materials characterization, (iv) material property evaluation: study of the performance of materials under service conditions, (v) materials design: materials selection, applications and quality control of manufactured products and (vi) failure analysis: investigating cause of failure.

Metals have been attractive for usage in daily life for centuries. Earliest known metals to mankind include iron, gold, copper, silver and zinc. Steel - a marvel on its own has been in extensive use for applications ranging from a simple blade to complex space components. Metals are easy to extract process and work with. The capability of metals to form alloys (without much economical expenditure) is valuable. The ease of processing of metals/alloys into varied shapes is a major advantage. Titanium, aluminium, magnesium and nickel metals/alloys have unique properties, and thereby they are in demand for critical applications. Aluminium and magnesium alloys have the lowest density amongst the structural metals and provide high specific strength properties. Titanium being light weight also exhibits excellent strength, corrosion resistance, fatigue resistance etc, and is a good candidate material for space/aerospace applications. Nickel and cobalt-based alloys have very high temperature stability and have ultra-high strength (superalloys). With the advancement in technology, the capability to create new advanced materials has increased multi-fold.

Ceramics and polymers/plastics have broad applications. Ceramics are high strength materials (e.g. $\mathrm{SiC}, \mathrm{Al}_{2} \mathrm{O}_{3}, \mathrm{~B}_{4} \mathrm{C}$ etc.) which are formed by ionic bonds. This renders them with high strength and excellent thermal stability. Polymers are easy to work with. They are cost-effective and can be processed into simple and also complex/intricate shapes through a variety of processing methods. In the past decades, concerted research world-wide has been focussed towards reinforcing metals/alloys, ceramics and polymers with various ceramics/glass/carbon materials to further improve their properties and performance. These are termed as 'composite materials', viz, metal matrix composites (MMCs), ceramic matrix composites (CMCs) and polymer matrix composites (PMCs). With such a possibility, properties of low strength metal/alloys and polymers have been improved to a great extent.

Processing of materials has evolved significantly over the decades. From basic metal casting to rapid solidification methods that are used to make amorphous/quasicrystalline/ nanocrystalline materials, the technology of materials processing has grown at a stupendous rate. Advanced processes to shape, form (near-net shape/micro/nano-forming) and finish (superfinish/mirror-finish) have been developed, paving way for the creation of new materials for advanced technologies.

In day-to-day life, some applications require materials that inherently possess specific/particular properties and functions called 'functional materials'. For example, the inherent ferromagnetic property of materials such as those based on $\mathrm{Fe}, \mathrm{Ni}$, Co, AlNiCo metallic and amorphous alloys (magnetic materials) is exploited in memory storage devices. Another example is that of graphitic carbon nanofibers consisting of stacks of graphene layers, which due to their unique electrical conductive surface are used as active catalytic materials for applications involving reactions.

Nanotechnology has brought forth nano-materials that have morphological features at nano-metre scale. These materials possess unique/special properties and capabilities due to their nano-scale dimensions. Nano-materials have found their niche is wide range of applications that include surface science, colloidal science, functional coatings, opto-electronics, energy materials, micro/nano-electronics, superconductivity, catalysis, energy storage systems, solar cells, biomedical devices, bioengineering and drug delivery. By the virtue of the properties of nano- 
materials, new/advanced devices have been realized, that were unattainable earlier with conventional materials.

In recent times, research in materials science/engineering is being put to use in developing materials for sustainable environment and renewables/alternative energy, such as water purification, energy efficient buildings, fuel cells, hydrogen storage materials, supercapacitors for photovoltaics and batteries, low carbon technologies.

Given the fact that enormous advancement in materials science and engineering has been/is being achieved at a rapid pace, it is envisioned that future technologies will revolutionize our daily life and thinking. 